\title{
TRIANGULATING NONSIMPLY CONNECTED MANIFOLDS
}

\author{
BY JOHN HOLLINGSWORTH ${ }^{1}$
}

Communicated by William Browder, January 30, 1969

Lashof and Rothenberg have recently announced the following

THEOREM. Let $M^{n}$ be a compact topological manifold with boundary $N^{n-1}$, with fundamental group satisfying condition $S$.

(a) If $H^{4}\left(M ; Z_{2}\right)=H^{3}\left(N ; Z_{2}\right)=0$, and $n \geqq 6, M$ admits a $P L$ manifold structure.

(b) If $N$ already has a PL structure, $H^{4}\left(M ; Z_{2}\right)=H^{3}\left(N ; Z_{2}\right)=0$ and $n \geqq 5$, then $M$ admits a $P L$ manifold structure agreeing with the given one on the boundary.

The condition $S$ is that $\pi_{1}\left(M \times T^{k}\right)$ and $\pi_{1}\left(\partial M \times T^{k}\right)$ satisfy the necessary conditions for the splitting theorems to hold, where $T^{k}$ is the $k$-torus. If $\pi_{1}(M)$ and $\pi_{1}(\partial M)$ are free abelian, then condition $S$ is satisfied. The purpose of this note is to relax the condition on the fundamental group.

THEOREM 1. Let $M^{n}$ be a closed, orientable topological manifold of dimension $n \geqq 7$ with $H^{4}\left(M ; Z_{2}\right)=0$. Then $M$ has a $P L$ structure.

Proof. By [1] and [2] or by [3], the stable homeomorphism conjecture is true in these dimensions, so $M$ has a stable structure. By [4], $\pi_{1}(M)$ is generated by imbedded one spheres with product neighborhoods. Let $f_{i}: S_{i}^{1} \times D^{n-1} \rightarrow M$ be such imbeddings, $i=1,2$,

$\cdots, k$. We may assume that the $f_{i}\left(S_{i}^{1} \times D^{n-1}\right)$ 's are disjoint. Let $0<\alpha<1$ and $D_{\alpha}^{n-1}=\left\{x \in R^{n-1} \mid\|x\| \leqq \alpha\right\}$. Henceforth we ignore the $f_{i}$ and consider $S_{\imath}^{l} \times D_{\alpha}^{n-1} \subset S_{\imath}^{1} \times D^{n-1} \subset M$.

Let

$$
\bar{M}=M-\bigcup_{i=1}^{k}\left(\operatorname{int}\left(S_{i}^{1} \times D_{\alpha}^{n-1}\right)\right)
$$

and

$$
M^{\prime}=\bar{M} \cup \bigcup_{i=1}^{k}\left(D_{i}^{2} \times S^{n-2}\right)
$$

That is, perform surgery on $M$ to kill $\pi_{1}(M)$. Then $\pi_{1}\left(M^{\prime}\right)=0$ and $H^{4}\left(M^{\prime} ; Z_{2}\right)=0$. By Lashof and Rothenberg, $M^{\prime}$ has a PL structure. Let $V=M-\bigcup_{i=1}^{k}\left(S_{i}^{1} \times D_{\alpha}^{n-1}\right)=M^{\prime}-\bigcup_{i=1}^{k} \quad\left(D_{i}^{2} \times S^{n-2}\right)$.

1 This work was partially supported by NSF Grant GP-8615. 
Then $V$ is an open subset of $M^{\prime}$, so $V$ has a PL structure. Let $\epsilon_{i}$ be the end of $V$ contained in $S_{i}^{1} \times D^{n-1}$. Then $\pi_{1}\left(\epsilon_{i}\right)=Z$, so by Siebenman, $\epsilon_{i}$ has a connected PL manifold neighborhood $N_{i}$ with $N_{i}$ $\cong \partial N_{i} \times[0,1)$. Let $W_{i}=N_{i} \cup \partial\left(S_{i}^{1} \times D_{\alpha}^{n-1}\right)$. Then $W_{i}$ is an $h$-cobordism between $\partial N_{i}$ and $\partial\left(S_{i}^{1} \times D_{\alpha}^{n-1}\right) \cong S^{1} \times S^{n-2}$, and hence $W_{i}$ satisfies the hypothesis of the theorem of Lashof-Rothenberg, and we extend the triangulations on $\partial N_{i}$ and $\partial\left(S_{i}^{1} \times D_{\alpha}^{n-1}\right)$ to all of $W_{i}$. Doing this for each $i=1,2, \cdots, k$, we get a triangulation for $M$, the triangulation induced from $M^{\prime}$ on $V-U N_{i}$, the triangulation of $W_{i}$, and the natural triangulation on $S_{i}^{1} \times D_{\alpha}^{n-1}$.

THEOREM 2. Let $M^{n}$ be a compact orientable manifold with boundary $N^{n-1}$ and suppose $H^{4}\left(M ; Z_{2}\right)=H^{3}\left(N ; Z_{2}\right)=0$. Then any $P L$ structure on $N$ extends to a $P L$ structure on $M$, provided $n \geqq 8$.

Proof. The proof is essentially the same as the proof of Theorem 1. As before $\pi_{1}(N)$ is generated by imbedded 1-spheres with product neighborhoods. Since $N$ is a PL manifold, we may assume that the imbeddings $f_{i}: S_{i}^{1} \times D^{n-2} \rightarrow N$ are piecewise linear. We use the maps $f_{i} \mid S_{i}^{1} \times D^{n-2}$ to attach handles $D_{i}^{2} \times D_{\alpha}^{n-2}$ to $M$. Call the resulting manifold $M_{1}$. Then $\partial M_{1}$ is a PL manifold with trivial fundamental group. We now perform surgeries on $M_{1}$ as in the proof of Theorem 1 to get $M^{\prime}$. Then $\pi_{1}\left(M^{\prime}\right)=\pi_{1}\left(\partial M^{\prime}\right)=0, H^{4}\left(M^{\prime} ; Z_{2}\right)=H^{3}\left(\partial M^{\prime} ; Z_{2}\right)=0$, so by Lashof and Rothenberg, $M^{\prime}$ has a PL structure agreeing with the given PL structure on $\partial M^{\prime}$. Just as in Theorem $1, M_{1}$ then has a PL structure agreeing with the given PL structure of $\partial M_{1}=\partial M^{\prime}$.

Let $W=M-\cup\left(f_{i}\left(S_{i}^{1} \times D_{\alpha}^{n-2}\right)\right)$. Then $W$ is an open subset of $M_{1}$, and so $W$ is a PL manifold. Let $\epsilon_{i}$ be the end of $W$ contained in a neighborhood of $f_{i}\left(S_{i}^{1} \times D_{\alpha}^{n-2}\right)$. Then $\epsilon_{i}$ is tame and $\pi_{1}\left(\epsilon_{i}\right)=Z$. By the relative Siebenman theorem, $\epsilon_{i}$ is collared. That is, there is a connected PL manifold neighborhood $V_{i}$ of $\epsilon_{i}$ such that $V_{i}$ is closed in $W$, frontier of $V_{i}$ is compact submanifold of $W$, and $V_{i} \cong \partial V_{i} \times[0,1)$, $V_{i} \cap \partial W \cong \partial\left(V_{i} \cap \partial W\right) \times[0,1)$. Let $U_{i}=V_{i} U_{f_{i}}\left(S_{i}^{1} \times D_{\alpha}^{n-2}\right)$. Then $U_{i}$ is a compact topological manifold with a PL triangulation on $\partial U_{i}$. (The triangulations on $\partial V_{i}$ and $\partial M$ agree on the $\partial V_{i} \cap \partial M_{i}$ ). Now $\pi_{1}\left(U_{i}\right)=Z, H^{4}\left(U_{i} ; Z_{2}\right)=H^{3}\left(\partial U_{i} ; Z_{2}\right)=0$, so by Lashof and Rothenberg, the triangulation on $\partial U_{i}$ extends to a PL triangulation of $U_{i}$. Doing this for each $i$ we get a PL structure on $M$ that agrees with the given PL structure on $\partial M$.

I have been told that R. C. Kirby and/or L. C. Siebenman have proved a stronger result independently and previously. Theorems 1 and 2 may still be of interest, however, in that the proofs remain 
valid without the condition on the cohomology groups, provided one can remove this condition in the theorem of Lashof-Rothenberg.

\section{BIBLIOGRAPHY}

1. R. C. Kirby, Stable homeomorphisms (to appear).

2. W. C. Hsiang and J. L. Shaneson, Fake tori and the annulus conjecture, Proc. Nat. Acad. Sci. U.S.A. 62 (1969).

3. R. Lashof and M. Rothenberg, Triangulation of manifolds. I, II, Bull. Amer. Math. Soc. 75 (1969), 750-754, 755-757.

4. M. Brown and H. Gluck, Stable homeomorphisms on manifolds. I, II, III, Ann. of Math. (2) 79 (1964), 1-58.

5. L. C. Siebenmann, The obstruction to finding a boundary for an open manifold of dimension greater than five, Ph.D. Thesis, Princeton University, Princeton, N.J., 1965.

6. R. Kirby and L. Siebenmann, On the triangulation of manifolds and the Hauptvermutung, Bull. Amer. Math. Soc. 75 (1969), 742-749.

University of Georgia, Athens, Georgia 30602 